\title{
Divided right atrium. Diagnosis by echocardiography, and considerations on the functional role of the Eustachian valve
}

\author{
Antonio F. Corno, Cédric Bron and Ludwig K. von Segesser \\ Pediatric Cardiac Surgery, Centre Hospitalier Universitaire Vaudois, Lausanne, Switzerland
}

\begin{abstract}
A child presented at birth with severe cyanosis. Echocardiography showed hypoplasia of the right heart with a right-to-left shunt at atrial level. A conservative approach was adopted initially, and the situation improved over a few months, with reversal of the atrial shunt. Surgery was successfully performed at 4 years of age after further echocardiography revealed a congenitally large Eustachian valve and an atrial septal defect.
\end{abstract}

Keywords: Hypoplastic right ventricle; Eustachian valve; right-to-left interatrial shunt

In the foetus, the systemic venous return of the lower part of the body is deviated directly to the left atrium through the oval foramen. This is facilitated by the existence of a fibrous flap separating the venous from the muscular part of the atrium. ${ }^{1}$ During the second trimester, this structure regress, leaving two remnants in the normal heart: the Thebesian and Eustachian valves. The valve can persist in part or more extensively, taking different shapes as previously described. ${ }^{2}$ Such division of the right atrium, usually called 'cor triatriatum dexter' or 'persistent right valve of the sinus venosus', ${ }^{2}$ is rarely diagnosed during life unless associated with obstruction of the usual pathway of blood to the right ventricle, or with other anomalies on the right heart. ${ }^{3}$ Rarely the only manifestation is a supraventricular arrhythmia. When this entity is not part of a complex malformation, surgery with good longterm prognosis can be contemplated, simply by removing the valvar remnant.

\section{Case report}

In a neonate with severe cyanosis (percutaneous saturation of oxygen in room air of $75 \%$ ), echo-

Correspondence to: Antonio F. Corno, MD, FRCS, Centre Hospitalier Universitaire Vaudois, Rue du Bugnon 46, CH-1011, Lausanne, Switzerland. Tel: 41213142280 ; Fax: 41213142278 ; E-mail: Antonio-Corno@chuv. hospvd.ch

Accepted for publication 2 November 1998 cardiography showed hypoplasia of the right heart, with a small dysplastic tricuspid valve, a small pulmonary valve with a diameter of $6 \mathrm{~mm}$ and an atrial septal defect in the oval fossa permitting a predominant right-to-left shunt. Conservative treatment was instituted, with administration of oxygen and preparation to give prostaglandin E1 should there be no improvement. During the first weeks of life, a progressive reduction of cyanosis was noted. At 4 months of age, the percutaneous saturation of oxygen had increased to $86 \%$, and the shunt at atrial level became bi-directional. The cyanosis continued to diminish, disappearing almost completely with time. At 4 years of age, the patient returned with normal growth on physical examination, was well perfused and did not have signs of heart failure. The peripheral pulses were all present and normal. Auscultation revealed a 2/6 systolic murmur at upper left sternal edge and fixed splitting of the second heart sound.

Systemic saturation of oxygen was $93 \%$ in room air. The electrocardiogram was normal, and chest $\mathrm{X}$-ray showed mild cardiomegaly without pulmonary plethora. Echocardiography showed a fenestrated defect in the oval fossa permitting a predominant left-to-right shunt, a dysplastic tricuspid valve of normal diameter $(19 \mathrm{~mm})$ with mild regurgitation and a very large Eustachian valve. The persistent Eustachian valve had been not seen in the previous investigations, but now moved back and forth between the right atrium 
and the right ventricle (Fig. 1) albeit without producing any obstruction to the blood flow through the tricuspid valve, and without generating any pressure gradient. The right ventricle showed no evidence of volume overload and had normal contractility. It was decided to remove the persistent venous valve. On normothermic cardiopulmonary bypass, the atrial septal defect was closed and the huge Eustachian valve was resected. The pathological report, describing a thick valvar structure with multiple small perforations and two cords, confirmed the diagnosis of persistence of the Eustachian valve (Fig. 2). Eight days post-operation the girl was discharged without any residual intracardiac shunt and with normal function of the tricuspid valve and right ventricle. She remains asymptomatic 2 years post-operation.

\section{Discussion}

Generally, when faced with a severely cyanotic neonate with hypoplastic right ventricle and dominant right-to-left shunt, there is little place for conservative treatment, a surgical approach usually being needed depending on the specific diagnosis. In the case presented here the choice for expectant treatment was based on the anticipated decrease in pulmonary vascular resistance, with its effects on the function and size of the right ventricle. If adequate size and contractility of the right ventricle can be obtained with time, simple closure of the atrial septal defect is curative. ${ }^{5}$

In addition to the valves of the embryonic venous sinus, the pulmonary resistance, very high during foetal life, helps oxygenated blood coming from the inferior caval vein to bypass the right ventricle and lungs via the oval foramen. Immediately after birth, pulmonary resistance lowers progressively, reaching its nadir at 3 months. In our case, it was thought that the flow of blood through the right ventricle would increase as those resistances were falling. Increasing the amount of blood reaching the right ventricle then prompted good development of the cavity of the right ventricle, which had not been associated with either tricuspid or pulmonary atresia, those being the commonest anomalies associated with such hypoplasia. ${ }^{6}$ With time and the decrease of the pulmonary vascular resistance, flow to the lungs increased, as did the pressure in the left atrium. At this time, the interatrial communication became of greater importance than the Eustachian valve itself because of supplementary blood flow returning through the atrial defect to the right ventricle.

In our patient, therefore, the conservative attitude, with simple observation and administration of oxygen, permitted adequate development of the right ventricle in term of size and function.

Based on experience with this patient, it is suggested that, when the right ventricle is seen to be hypoplastic at birth, the potential significance of an extensively persistent Eustachian valve preventing flow of blood through the tricuspid valve to the right ventricle should be ruled out by echocardiography.

The resection of the Eustachian valve, associated with closure of the atrial septal defect once the decrease of pulmonary vascular resistance and the development of the right ventricle has reversed the direction of the neonatal right-to-left interatrial
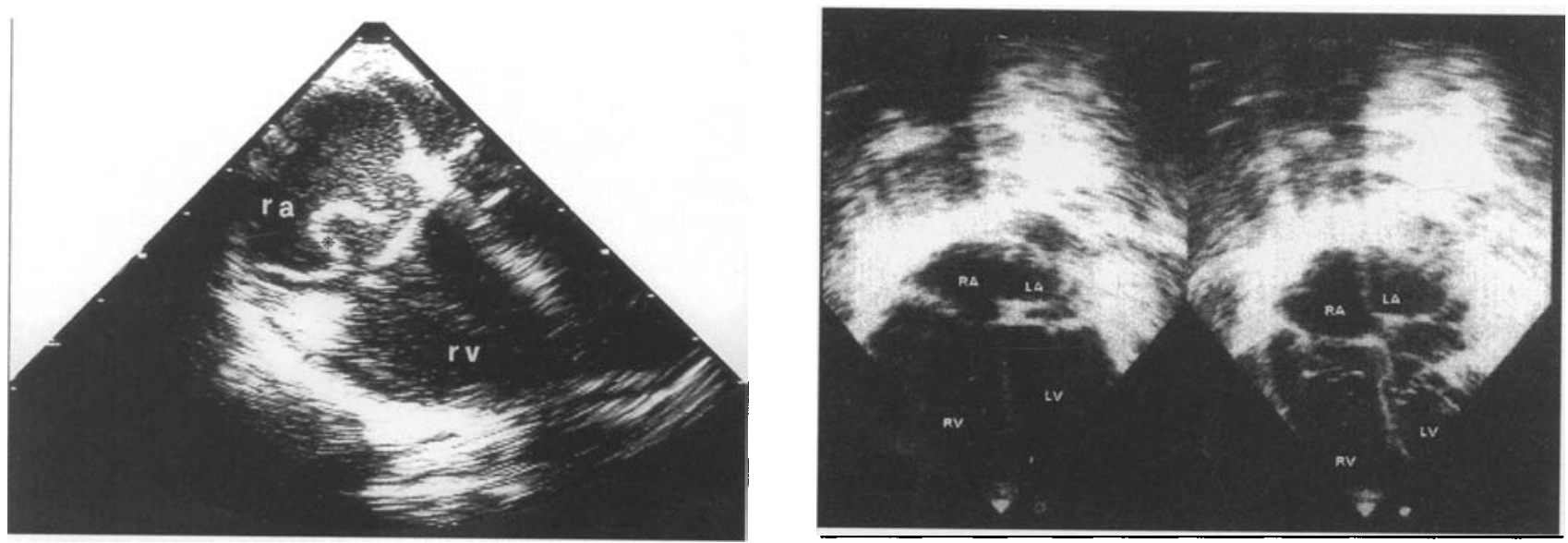

Figure 1.

Pre-operative transoesophagal echocardiography showing the Eustachian valve $\left(^{*}\right)$ in the atrium during diastole (a) and during systole (b), in the proximity of the tricuspid valve. ra, Right atrium; $w$, right ventricle. 


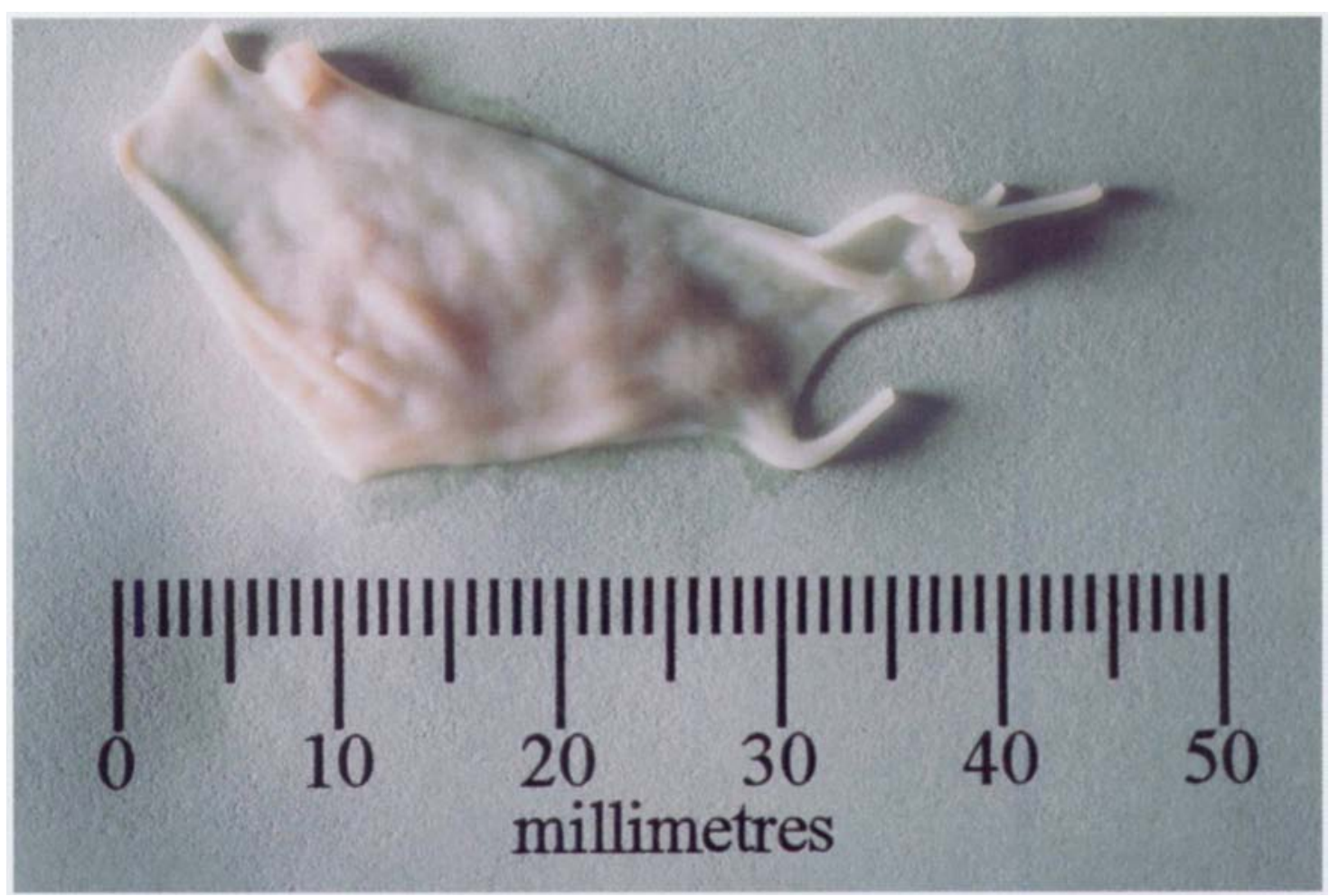

Figure 2.

Surgically resected abnormal Eustachian valve.

shunt, is self-evidently a surgical option preferable to any other, such as neonatal construction of a systemic-to-pulmonary artery shunt, or any other step towards an univentricular type of repair.

\section{References}

1. Anderson RH. Understanding the nature of congenital division of the atrial chambers. Br Heart J 1992; 68: 1-3.

2. Doucette J, Knoblich R. Persistent right valve of the sinus venosus. Arch Pathol 1963; 75: 117-123.
3. Trento A, Zuberbuhler JR, Anderson RH, Park SC, Sewers RD. Divided right atrium (prominence of the Eustachian and Thebesian valves). J Thorac Cardiovasc Surg 1988; 96: 457-463.

4. Ott DA, Cooley DA, Angelini P, Leachman RD. Successful surgical correction of symptomatic cor atrium dexter. $J$ Thorac Cardiovasc Surg 1979; 78: 573-575.

5. Goh K, Sasajima T, Inaba M, Yamamoto H, Kawashima E, Kubo Y. Isolated right ventricular hypoplasia: intraoperative balloon occlusion test. Ann Thorac Surg 1998; 65: 551-553.

6. Becker AE, Anderson RH. Hypoplasia of the right ventricle. In Pathology of Congenital Heart Disease. Butterworths, London, 1981, pp 119-127. 